\title{
POLYNOMIAL VALUES OF SUMS OF PRODUCTS OF CONSECUTIVE INTEGERS
}

\author{
A. BAZSÓ, A. BÉRCZES, L. HAJDU, F. LUCA \\ Dedicated to Professor Ákos Pintér on the occasion of his 50th birthday.
}

\begin{abstract}
We investigate polynomial values of sums of products of consecutive integers. For the degree two case we give effective finiteness results, while for the higher degree case we provide ineffective finiteness theorems. For the latter purpose, we also show that the polynomials corresponding to the sums of products we investigate, are indecomposable.
\end{abstract}

\section{INTRODUCTION}

The polynomial values of combinatorial polynomials have a vast literature. Here we only mention the papers $[2,5,8,10]$, and the references given there. In this paper, we consider the problem of describing the polynomial values of a family of polynomials related to the sums of products of consecutive integers.

For $k=0,1,2, \ldots$ put

$$
f_{k}(x)=\sum_{i=0}^{k} \prod_{j=0}^{i}(x+j) .
$$

The first few such polynomials are:

$$
\begin{gathered}
f_{0}(x)=x, f_{1}(x)=x+x(x+1)=x(x+2), \\
f_{2}(x)=x+x(x+1)+x(x+1)(x+2)=x(x+2)^{2}, \\
f_{3}(x)=x(x+2)\left(x^{2}+5 x+5\right), f_{4}(x)=x(x+2)\left(x^{3}+9 x^{2}+24 x+17\right) .
\end{gathered}
$$

In general, $f_{k}(x)$ is a monic polynomial with positive integer coefficients and of degree $k+1$.

These polynomials were introduced by Hajdu, Laishram and Tengely [9], who considered their power values, i.e. the Diophantine equation

$$
f_{k}(x)=y^{n} \text {. }
$$

Date: April 2, 2017.

2010 Mathematics Subject Classification. 11D41.

Key words and phrases. Sums of products, blocks of consecutive integers, polynomial values. 
They proved effective finiteness results on the above equation, and they gave all solutions when $1 \leq k \leq 10$ such that $k \neq 2$ if $n=2$. Along the way, they also proved that for $k \geq 3$, all the roots of $f_{k}(x)$ are real and simple. We mention that this problem is related to several classical questions; e.g., to the power values of products of consecutive integers (see the fundamental paper of Erdős and Selfridge [7]). For a more precise account, we refer to the paper [9].

The structure of the paper is the following. In the next section, we give our main results. Then we formulate some lemmas and auxiliary results. Finally, we prove our statements in separate sections.

\section{NeW RESUlts}

Let $g(x) \in \mathbb{Q}[x]$ be an arbitrary polynomial, and consider the Diophantine equation

$$
f_{k}(x)=g(y) \quad(x, y \in \mathbb{Z}) .
$$

Our first result gives a general (partly effective) finiteness theorem for (1).

Theorem 1. Let $k \geq 3$. (i) For $\operatorname{deg} g \in\{0,2\}$, there exist an effectively computable constant $C_{1}(k, g)$ depending only on $k$ and $g$ such that $\max (|x|,|y|)<C_{1}(k, g)$ for each integer solutions of equation (1).

(ii) For $\operatorname{deg} g \geq 3$, equation (1) has only finitely many integer solutions $x, y$, unless we have $g(x)=f_{k}(h(x))$, where $h(x) \in \mathbb{Q}[x]$ with $\operatorname{deg}(h)>0$.

Clearly, in the exceptional cases $\operatorname{deg} g=1$ and $g(x)=f_{k}(h(x))$, equation (1) can have infinitely many integer solutions $x, y$. Further, in the cases $k \leq 2$ equation (1) can also have infinitely many solutions, which can be described easily.

We note that in the proof of part (ii) of our Theorem 1 we use the ineffective finiteness criterion of Bilu and Tichy [4] combined with Theorem 3. Thus, part (ii) is an ineffective statement.

Consider now the equation

$$
f_{k}(x)=a y^{n}+b,
$$

in integers $x, y, n$ with $n \geq 2$, where $a, b, k$ are given integers with $k \geq 0$. The following theorem extends Theorem 2.1. of [9], where only the case $a=1, b=0$ has been considered.

Theorem 2. For $k \geq 3$, we have $\max (|x|,|y|)<C_{2}(k, a, b)$ for each integer solutions of equation (2). Further, if $|y|>1$, then we have $n<$ $C_{3}(k, a, b)$. Here, $C_{2}(k, a, b)$ and $C_{3}(k, a, b)$ are effectively computable constants depending only on $k, a, b$. 
Note that our second assumption is necessary for bounding $n$ in (2): obviously, $n$ cannot be bounded in the case $y \in\{-1,0,1\}$.

Finally, we prove the indecomposability of the polynomial $f_{k}(x)$. This plays an important role in the proof of part (ii) of Theorem 1 . By a decomposition of a polynomial $F(x)$ over a field $\mathbb{K}$ we mean writing $F(x)$ as

$$
F(x)=G_{1}\left(G_{2}(x)\right) \quad\left(G_{1}(x), G_{2}(x) \in \mathbb{K}[x]\right),
$$

which is nontrivial if

$$
\operatorname{deg} G_{1}(x)>1 \quad \text { and } \quad \operatorname{deg} G_{2}(x)>1 .
$$

Two decompositions $F(x)=G_{1}\left(G_{2}(x)\right)$ and $F(x)=H_{1}\left(H_{2}(x)\right)$ are said to be equivalent if there exists a linear polynomial $\ell(x) \in \mathbb{K}[x]$ such that $G_{1}(x)=H_{1}(\ell(x))$ and $H_{2}(x)=\ell\left(G_{2}(x)\right)$. The polynomial $F(x)$ is called decomposable over $\mathbb{K}$ if it has at least one nontrivial decomposition over $\mathbb{K}$; otherwise it is said to be indecomposable.

Theorem 3. The polynomial $f_{k}(x)$ is indecomposable over $\mathbb{C}$ for any $k \geq 0$.

\section{LEMMAS AND AUXILIARY RESULTS}

In this section, we give some results needed to prove our theorems. First, we recall the finiteness criterion of Bilu and Tichy [4]. To do this, we need to define five kinds of so-called standard pairs of polynomials.

Let $\alpha, \beta, \delta$ be nonzero rational numbers, $\mu, \nu, q>0$ and $r \geq 0$ be integers, and let $v(x) \in \mathbb{Q}[x]$ be a nonzero polynomial (which may be constant). Denote by $D_{\mu}(x, \delta)$ the $\mu$-th Dickson polynomial, given by

$$
D_{\mu}(x, \delta)=\sum_{i=0}^{\lfloor\mu / 2\rfloor} d_{\mu, i} x^{\mu-2 i} \quad \text { with } \quad d_{\mu, i}=\frac{\mu}{\mu-i}\left(\begin{array}{c}
\mu-i \\
i
\end{array}\right)(-\delta)^{i} .
$$

For properties of Dickson polynomials, we refer to [11].

Two polynomials $F(x)$ and $G(x)$ are said to form a standard pair over $\mathbb{Q}$ if one of the ordered pairs $(F(x), G(x))$ or $(G(x), F(x))$ belongs to the list below. The five kinds of standard pairs are then listed in Table 1.

Now we state a special case of the main result of [4], which will be crucial in the proof of Theorem 1 (ii).

Lemma 1. Let $f(x), g(x) \in \mathbb{Q}[x]$ be nonconstant polynomials such that the equation $f(x)=g(y)$ has infinitely many solutions in rational integers $x, y$. Then $f=\varphi \circ F \circ \lambda$ and $g=\varphi \circ G \circ \kappa$, where $\lambda(x), \kappa(x) \in \mathbb{Q}[x]$ are linear polynomials, $\varphi(x) \in \mathbb{Q}[x]$, and $F(x), G(x)$ form a standard pair over $\mathbb{Q}$. 


\begin{tabular}{|c|c|c|}
\hline kind & standard pair & parameter restrictions \\
\hline first & $\left(x^{q}, \alpha x^{r} v(x)^{q}\right)$ & $0 \leq r<q,(r, q)=1$, \\
& & $r+\operatorname{deg} v(x)>0$ \\
\hline second & $\left(x^{2},\left(\alpha x^{2}+\beta\right) v(x)^{2}\right)$ & - \\
\hline third & $\left(D_{\mu}\left(x, \alpha^{\nu}\right), D_{\nu}\left(x, \alpha^{\mu}\right)\right)$ & $(\mu, \nu)=1$ \\
\hline fourth & $\left(\alpha^{\frac{-\mu}{2}} D_{\mu}(x, \alpha),-\beta^{-\frac{\nu}{2}} D_{\nu}(x, \beta)\right)$ & $(\mu, \nu)=2$ \\
\hline fifth & $\left(\left(\alpha x^{2}-1\right)^{3}, 3 x^{4}-4 x^{3}\right)$ & - \\
\hline
\end{tabular}

TABLE 1. Standard pairs

Let $f(x) \in \mathbb{Z}[x]$ be a nonzero polynomial of degree $d$ and height $H$. Further, let $a$ be a nonzero integer. Consider the Diophantine equation

$$
f(x)=a y^{n} .
$$

The next lemma is a special case of a result of Bérczes, Brindza and Hajdu [1]. For the first results of this type, we refer to [12] and [13].

Lemma 2. If $f(x)$ has at least two distinct roots and $|y|>1$, then, in (3), we have $n<C_{4}(d, H, a)$, where $C_{4}(d, H, a)$ is an effectively computable constant depending only on $d, H$ and $a$.

The following result is a special case of an effective theorem of Brindza [3].

Lemma 3. If, in (3), either $n=2$ and $f(x)$ has at least three zeros of odd multiplicities, or $n \geq 3$ and $f(x)$ has at least two zeros of multiplicities coprime to $n$, then for each solutions of (3) we have $\max (|x|,|y|)<C_{5}(d, H, a)$, where $C_{5}(d, H, a)$ is an effectively computable constant depending only on $d, H$ and $b$.

We recall Lemma 3.3 from [9], which describes the root structure of the polynomial family $f_{k}(x)$.

Lemma 4. We have $f_{0}(x)=x, f_{1}(x)=x(x+2), f_{2}(x)=x(x+2)^{2}$. Besides this, for every $k \geq 3$, all the roots of the polynomial $f_{k}(x)$ are real and simple. In particular, 0 is a root of $f_{k}(x)$ for all $k \geq 0$, and -2 is a root of $f_{k}(x)$ for all $k \geq 1$. Moreover, for $k \geq 3, f_{k}(x)$ has a root in each of the following intervals:

$(-1,1),(-1.5,-1),(-3,-1.5),(-4,-3),(-5,-4), \ldots,(-k-1,-k)$.

In the next three lemmas we make some observations on the roots of the derivative $f_{k}^{\prime}(x)$ of the polynomial $f_{k}(x)$.

Lemma 5. For $k \geq 3$, all the roots of the polynomial $f_{k}^{\prime}(x)$ are real and simple. 
Proof. This follows from Lemma 4 by Rolle's theorem.

In what follows, we use the following notation. For $i \geq 0$, we let $P_{i}(x)=x(x+1) \cdots(x+i)$. Then, $f_{k}(x)=P_{0}(x)+P_{1}(x)+\cdots+P_{k}(x)$.

Lemma 6. For $k \geq 6$, we have $f_{k}^{\prime}(0)>0, f_{k}^{\prime}(-1)<0, f_{k}^{\prime}(-2)>0$, $f_{k}^{\prime}(-3)<0, f_{k}^{\prime}(-4)>0, f_{k}^{\prime}(-5)<0$.

Proof. Observe that

(4) $f_{k}^{\prime}(x)=\sum_{i=0}^{k} P_{i}^{\prime}(x)=\sum_{i=0}^{k} \sum_{j=0}^{i} \frac{P_{i}(x)}{x+j}$

$$
\begin{array}{r}
=1+[(x+1)+x]+[(x+1)(x+2)+x(x+2)+x(x+1)] \\
+[(x+1)(x+2)(x+3)+x(x+2)(x+3)+x(x+1)(x+3) \\
+x(x+1)(x+2)]+\cdots
\end{array}
$$

holds for each $k \geq 0$, whence $f_{k}^{\prime}(0)>0$ immediately follows.

By considering the first few summands of (4), we obtain

$$
\begin{aligned}
& f_{k}^{\prime}(-1)=P_{0}^{\prime}(-1)+P_{1}^{\prime}(-1)+P_{2}^{\prime}(-1)+P_{3}^{\prime}(-1)+\cdots \\
& =1+[-1]+[(-1)(-1+2)]+[(-1)(-1+2)(-1+3)]+\cdots .
\end{aligned}
$$

We infer that $P_{i}^{\prime}(-1)<0$ for $i \geq 2$, so $f_{k}^{\prime}(-1)<0$ if $k \geq 2$.

Similarly,

(6) $f_{k}^{\prime}(-2)=P_{0}^{\prime}(-2)+P_{1}^{\prime}(-2)+P_{2}^{\prime}(-2)+P_{3}^{\prime}(-2)+\cdots$

$$
=1+[-1-2]+[(-2)(-2+1)]+[(-2)(-2+1)(-2+3)]+\cdots
$$

implies that $P_{i}^{\prime}(-2)>0$ for $i \geq 2$, and that $f_{k}^{\prime}(-2)>0$ if $k \geq 3$.

Analogously one can observe that $f_{k}^{\prime}(-3)<0$ if $k \geq 4$; further that $f_{k}^{\prime}(-4)>0$ if $k \geq 5$; and that $f_{k}^{\prime}(-5)<0$ if $k \geq 6$.

Let $\alpha_{1}>\alpha_{2}>\cdots>\alpha_{k}$ denote the roots of $f_{k}^{\prime}(x)$. Clearly, $f_{k}(x)$ has a local extremum at each $\alpha_{i}$. Moreover, for $k \geq 6$, Lemmas 5 and 6 imply that

$$
0>\alpha_{1}>-1>\alpha_{2}>-2>\alpha_{3}>-3>\alpha_{4}>-4>\alpha_{5}>-5 .
$$

Further, let $\beta_{0}=0>\beta_{1}>\cdots>\beta_{k}$ denote the roots of $f_{k}(x)$. Obviously, $0=\beta_{0}>\alpha_{1}>\beta_{1}>\alpha_{2}>\beta_{2}>\cdots$. Moreover, for $k \geq 6$, Lemma 4 implies that

(8)

$$
0=\beta_{0}>-1>\beta_{1}>-\frac{3}{2}>-2=\beta_{2}>-3>\beta_{3}>-4>\beta_{4}>-5>\beta_{5} .
$$


Lemma 7. For $k \geq 13$ we have

$$
\left|f_{k}\left(\alpha_{1}\right)\right|>\left|f_{k}\left(\alpha_{3}\right)\right|>\left|f_{k}\left(\alpha_{5}\right)\right|
$$

and

$$
\left|f_{k}\left(\alpha_{2}\right)\right|>\left|f_{k}\left(\alpha_{4}\right)\right| .
$$

Proof. First, we show that the inequality $\left|f_{k}\left(\alpha_{1}\right)\right|>\left|f_{k}\left(\alpha_{3}\right)\right|$ holds. By (8), we have $\beta_{1}<-\frac{1}{2}<\beta_{0}$. Then

$$
\left|f_{k}\left(-\frac{1}{2}\right)\right| \leq\left|f_{k}\left(\alpha_{1}\right)\right| \text {. }
$$

On the other hand, for $k \geq 6,-3<\alpha_{3}<-2$ by (7), whence

$$
\left|f_{k}\left(\alpha_{3}\right)\right| \leq \max _{-3 \leq t \leq-2}\left|f_{k}(t)\right| \text {. }
$$

Observe that for any $i \geq 4, k \geq 6$ and $-3 \leq t \leq-2$, we have

$$
\left|P_{i}(t)\right| \leq 3 \cdot 2 \cdot \frac{1}{2} \cdot \frac{1}{2} \cdot 2 \cdot 3 \cdots(i-2)=\frac{3}{2}(i-2) ! .
$$

Furthermore, again for $i \geq 4$, we have

$$
\begin{aligned}
\left|P_{i}\left(-\frac{1}{2}\right)\right| & =\frac{1}{2} \cdot \frac{1}{2} \cdot \frac{3}{2} \cdot \frac{5}{2} \cdots \cdots \frac{2 i-1}{2} \\
& =\frac{2 i-1}{4^{i}} \cdot\left(\begin{array}{c}
2 i-2 \\
i-1
\end{array}\right) \cdot(i-1) ! \geq \frac{2 i-1}{8 \sqrt{i-1}} \cdot(i-1) ! .
\end{aligned}
$$

Here we used the well-known inequality

$$
\frac{4^{n}}{2 \sqrt{n}} \leq\left(\begin{array}{c}
2 n \\
n
\end{array}\right)
$$

which is valid for any $n>0$, and can be easily verified by induction.

Combining (11) - (14), we have

$$
\begin{aligned}
\left|f_{k}\left(\alpha_{1}\right)\right|- & \left|f_{k}\left(\alpha_{3}\right)\right| \geq\left|f_{k}\left(-\frac{1}{2}\right)\right|-\max _{-3 \leq t \leq-2}\left|f_{k}(t)\right| \\
\geq & \left|\sum_{i=0}^{3} P_{i}\left(-\frac{1}{2}\right)\right|-\sum_{i=0}^{3} \max _{-3 \leq t \leq-2}\left|P_{i}(t)\right| \\
& +\sum_{i=4}^{k}\left(\frac{(2 i-1) \sqrt{i-1}}{8}-\frac{3}{2}\right)(i-2) ! \\
& \geq-14+\sum_{i=4}^{k}\left(\frac{(2 i-1) \sqrt{i-1}}{8}-\frac{3}{2}\right)(i-2) !
\end{aligned}
$$

and the last expression above is positive for $k \geq 6$. 
Next, we verify the inequality $\left|f_{k}\left(\alpha_{3}\right)\right|>\left|f_{k}\left(\alpha_{5}\right)\right|$. Similarly as above, by (8), we notice that $\beta_{3}<-\frac{5}{2}<\beta_{2}$. Thus,

$$
\left|f_{k}\left(-\frac{5}{2}\right)\right| \leq\left|f_{k}\left(\alpha_{3}\right)\right|
$$

Besides, by (7), we have $-5<\alpha_{5}<-4$ for $k \geq 6$, whence

$$
\left|f_{k}\left(\alpha_{5}\right)\right| \leq \max _{-5 \leq t \leq-4}\left|f_{k}(t)\right|
$$

Observe now that for any $i \geq 6, k \geq 6$ and $-5 \leq t \leq-4$, we have

$$
\left|P_{i}(t)\right| \leq 5 \cdot 4 \cdot 3 \cdot 2 \cdot \frac{1}{2} \cdot \frac{1}{2} \cdot 2 \cdot 3 \cdots(i-4)=30(i-4) !,
$$

while, again for $i \geq 6$, we have, by (15), that

$$
\begin{aligned}
& \left|P_{i}\left(-\frac{5}{2}\right)\right|=\frac{5}{2} \cdot \frac{3}{2} \cdot \frac{1}{2} \cdot \frac{1}{2} \cdot \frac{3}{2} \cdot \frac{5}{2} \cdots \cdots \frac{2 i-5}{2} \\
& \quad=\frac{15(2 i-5)}{4^{i-1}} \cdot\left(\begin{array}{c}
2 i-6 \\
i-3
\end{array}\right) \cdot(i-3) ! \geq \frac{15(2 i-5)}{32 \sqrt{i-3}} \cdot(i-3) ! .
\end{aligned}
$$

From (17) - (20), we get

$$
\begin{aligned}
\left|f_{k}\left(\alpha_{3}\right)\right|- & \left|f_{k}\left(\alpha_{5}\right)\right| \geq\left|f_{k}\left(-\frac{5}{2}\right)\right|-\max _{-5 \leq t \leq-4}\left|f_{k}(t)\right| \\
& \geq\left|\sum_{i=0}^{5} P_{i}\left(-\frac{5}{2}\right)\right|-\sum_{i=0}^{5} \max _{-5 \leq t \leq-4}\left|P_{i}(t)\right| \\
& +\sum_{i=6}^{k}\left(\frac{15(2 i-5) \sqrt{i-3}}{32}-30\right)(i-4) ! \\
& \geq-336+\sum_{i=6}^{k}\left(\frac{15(2 i-5) \sqrt{i-3}}{32}-30\right)(i-4) !
\end{aligned}
$$

One can easily check that the last expression is positive when $k \geq 13$, whence $\left|f_{k}\left(\alpha_{3}\right)\right|>\left|f_{k}\left(\alpha_{5}\right)\right|$ holds for $k \geq 13$.

Finally, we prove (10). Similarly as before, we start by noticing that, by (8), $\beta_{2}<-\frac{3}{2}<\beta_{1}$, which yields

$$
\left|f_{k}\left(-\frac{3}{2}\right)\right| \leq\left|f_{k}\left(\alpha_{2}\right)\right| \text {. }
$$

On the other hand, since, by (7), $-4<\alpha_{4}<-3$ for $k \geq 6$, we have

$$
\left|f_{k}\left(\alpha_{4}\right)\right| \leq \max _{-4 \leq t \leq-3}\left|f_{k}(t)\right| \text {. }
$$


Let $i \geq 5, k \geq 6$ and $t \in(-4,-3)$. Then

$$
\left|P_{i}(t)\right| \leq 4 \cdot 3 \cdot 2 \cdot \frac{1}{2} \cdot \frac{1}{2} \cdot 2 \cdot 3 \cdots(i-3)=6(i-3) ! .
$$

By (15), we obtain, again for $i \geq 5$, that

$$
\begin{aligned}
\left|P_{i}\left(-\frac{3}{2}\right)\right| & =\frac{3}{2} \cdot \frac{1}{2} \cdot \frac{1}{2} \cdot \frac{3}{2} \cdot \frac{5}{2} \cdot \ldots \cdot \frac{2 i-3}{2} \\
& =\frac{3(2 i-3)}{2 \cdot 4^{i-1}} \cdot\left(\begin{array}{c}
2 i-4 \\
i-2
\end{array}\right) \cdot(i-2) ! \geq \frac{3(2 i-3)}{16 \sqrt{i-2}} \cdot(i-2) ! .
\end{aligned}
$$

Using (22) - (25), we obtain

$$
\begin{aligned}
\left|f_{k}\left(\alpha_{2}\right)\right|- & \left|f_{k}\left(\alpha_{4}\right)\right| \geq\left|f_{k}\left(-\frac{3}{2}\right)\right|-\max _{-4 \leq t \leq-3}\left|f_{k}(t)\right| \\
& \geq\left|\sum_{i=0}^{4} P_{i}\left(-\frac{3}{2}\right)\right|-\sum_{i=0}^{4} \max _{-4 \leq t \leq-3}\left|P_{i}(t)\right| \\
& +\sum_{i=5}^{k}\left(\frac{3(2 i-3) \sqrt{i-2}}{16}-6\right)(i-3) ! \\
& \geq-67+\sum_{i=5}^{k}\left(\frac{3(2 i-3) \sqrt{i-2}}{16}-6\right)(i-3) !
\end{aligned}
$$

We obtain that the last expression above is positive for $k \geq 9$. Thus, inequality (10) holds for $k \geq 9$, which completes the proof.

The following lemma is a slight modification of Lemma 3.3 of [9] and describes the root structure of the polynomials $f_{k}(x)+1$.

Lemma 8. For $k \geq 2$ all roots of the polynomial $f_{k}(x)+1$ are real and simple. Further, $f_{k}(x)+1$ has a root at -1 as well as a root in each the following $k$ intervals:

$$
(-k-1,-k),(-k,-k+1), \ldots,(-3,-2) \text { and }(-0.5,0) .
$$

Proof. The proof is just an adaptation of the proof of Lemma 3.3 of [9] to the polynomials $f_{k}(x)+1$. Clearly, $f_{k}(-1)=-1$ for any $k \in \mathbb{N}$, thus -1 is a root of $f_{k}(x)+1$. Since $f_{k}(0)+1=1$ and $f_{k}(-0.5)+1<0$, we see that the continuous function $f_{k}(x)+1$ has a zero in the interval $(-0.5,0)$.

It is easy to see that for any fixed $k \in \mathbb{N}$ and any $i=-2,-3, \ldots,-k$, $-k-1$, we have

$$
(-1)^{i}\left(f_{k}(i)+1\right)>0
$$


Thus, the polynomial $f_{k}(x)+1$ has a root in each of the intervals

$$
(-k-1,-k),(-k,-k+1), \ldots,(-3,-2) .
$$

This proves the lemma.

The next statement is due to Dujella and Gusić [6].

Lemma 9. Let $f(x) \in \mathbb{Z}[x]$ be monic and decomposable over $\mathbb{C}$. Then $f(x)$ is decomposable over $\mathbb{Z}$.

The following result is also due to Dujella and Gusić [6].

Lemma 10. Let $f(x)=x^{n}+a x^{n-1}+\cdots \in \mathbb{Z}[x]$ and suppose that $f(x)=G(H(x))$ for some monic polynomials $G(x), H(x) \in \mathbb{C}[x]$ with $\operatorname{deg} G=m$, $\operatorname{deg} H=k$. Then we have $m \leq \operatorname{gcd}(a, n)$. In particular, if $\operatorname{gcd}(a, n)=1$, then $f$ is indecomposable over $\mathbb{C}$.

Proof. The second statement is a theorem of Dujella and Gusić [6]. Its proof also implies the first statement, so for convenience we recall the proof from [6]. Assume that $f$ is decomposable over $\mathbb{C}$. Then, by Lemma $9, f$ is decomposable over $\mathbb{Z}$; i.e., there exists monic polinomials $G(x), H(x) \in \mathbb{Z}[x]$ such that $f(x)=G(H(x)), \operatorname{deg} G=m, \operatorname{deg} H=k$, $m, k \geq 2$. Since $f(x)=\left(x^{k}+c_{k-1} x^{k-1}+\ldots\right)^{m}+\cdots$ with $c_{k-1} \in \mathbb{Z}$, we have $n=m k$ and $a=m c_{k-1}$, which implies $\operatorname{gcd}(a, n) \geq m \geq 2$.

Now we start to investigate whether a linear transformation of $f_{k}(x)$ can be a linear transformation of a polynomial belonging to some standard pair.

Lemma 11. Let $c_{1}, c_{0}, e_{1}, e_{0} \in \mathbb{Q}$ with $c_{1} \neq 0$. Then the polynomial $f_{k}\left(c_{1} x+c_{0}\right)$ is not of the form $e_{1} x^{q}+e_{0}$ with $q \geq 3$.

Proof. Suppose that $f_{k}\left(c_{1} x+c_{0}\right)=e_{1} x^{q}+e_{0}$ with some fixed $q \geq 3$. Then clearly $e_{1} \neq 0$ and $q=k+1$. By Lemma 4, every root of the polynomial $f_{k}(x)$ is real, so all the roots of $f_{k}\left(c_{1} x+c_{0}\right)$ are also real. This is obviously not true for the polynomial $e_{1} x^{q}+e_{0}$ for $q \geq 3$. Thus, the desired conclusion follows.

Lemma 12. Let $c_{1}, c_{0}, e_{1}, e_{0} \in \mathbb{Q}$ with $c_{1} \neq 0$. Then the polynomial $f_{k}\left(c_{1} x+c_{0}\right)$ is not of the form

$$
e_{1} D_{\nu}(x, \delta)+e_{0}
$$

where $D_{\nu}(x, \delta)$ is the $\nu$-th Dickson polynomial with $\nu>3$ and $\delta \in$ $\mathbb{Q} \backslash\{0\}$.

Proof. Suppose that

$$
f_{k}\left(c_{1} x+c_{0}\right)=e_{1} D_{\nu}(x, \delta)+e_{0}
$$


holds for some $c_{1}, c_{0}, e_{1}, e_{0}$ and $\nu, \delta$ as in the statement. Then clearly $e_{1} \neq 0$ and $k+1=\nu$; in particular, $k \geq 3$. Equating the coefficients of $x^{k}$ in (27) we obtain

$$
c_{1}^{k}\left(1+\frac{(k+1)\left(k+2 c_{0}\right)}{2}\right)=0,
$$

which, since $c_{1} \neq 0$, implies that

$$
c_{0}=-\frac{k^{2}+k+2}{2(k+1)} .
$$

Similarly, comparing the coefficients of $x^{k-2}$ in (27), we obtain

$$
\begin{aligned}
& \frac{c_{1}^{k-2}}{48}\left(48+(k-1)\left(k^{5}+6 c_{0} k^{4}+\left(12 c_{0}^{2}+4 c_{0}+3\right) k^{3}+\right.\right. \\
& +\left(8 c_{0}^{3}+12 c_{0}^{2}+18 c_{0}-16\right) k^{2}+\left(8 c_{0}^{3}+24 c_{0}^{2}-28 c_{0}+28\right) k+ \\
& \left.\left.+48 c_{0}-48\right)\right)=0 .
\end{aligned}
$$

Substituting (29) into (30) we get

$$
\frac{k^{4}-2 k^{3}-5 k^{2}+12 k+18}{6(k+1)^{2}}=0,
$$

which implies that $k \notin \mathbb{Z}$, a contradiction.

\section{Proof of Theorem 3}

Put $f_{k}(x)=x^{k+1}+c_{k} x^{k}+c_{k-1} x^{k-1}+\cdots+c_{1} x$. Clearly, $c_{i} \in \mathbb{Z}$ for all $i$.

For the coefficient $c_{k}$ of $x^{k}$ in $f_{k}(x)$ we have $c_{k}=1+k(k+1) / 2$. If $k$ is even, then $\operatorname{gcd}\left(k+1, c_{k}\right)=1$ and by Lemma $10, f_{k}(x)$ is indecomposable. If $k$ is odd, then we have $\operatorname{gcd}\left(k+1, c_{k}\right) \leq 2$. Then, again by Lemma 10, it follows that if $f_{k}(x)$ is decomposable, then the outer polynomial of any decomposition has degree at most 2. Suppose that we have

$$
f_{k}(x)=a G(x)^{2}+b G(x)+c .
$$

By Lemma 9, we may suppose that $a, b, c$ are integers and $G(x) \in \mathbb{Z}[x]$. Then, clearly $a=1$. Let $u, v$ be the roots of the polynomial $y^{2}+b y+c$. Then we may write

$$
f_{k}(x)=(G(x)-u)(G(x)-v) .
$$

Since $f_{k}(0)=0$, we have either $G(0)=u$ or $G(0)=v$. Thus, one of $u$ and $v$ is an integer. Hence, both of $u, v$ are integers. We now put $H(x)=G(x)-u$. Then $H(x) \in \mathbb{Z}[x]$, and

$$
f_{k}(x)=H(x)(H(x)+d),
$$


where $d=u-v$. Observe that, by $f_{k}(-1)=-1, H(-1)= \pm 1$ and $H(-1)+d= \pm 1$, whence either $d=0$ or $d= \pm 2$. If $d=0$, then $f_{k}(x)=H(x)^{2}$, which contradicts Lemma 4. If $d= \pm 2$, then we have $f_{k}(x)+1=(H(x) \pm 1)^{2}$, which contradicts Lemma 8. Thus, $f_{k}(x)$ is indecomposable.

\section{Proof of Theorem 1 (ii)}

Let $k \geq 3$ and $g(x) \in \mathbb{Q}[x]$ be a polynomial with $\operatorname{deg} g \geq 3$. Suppose that equation (1) has infinitely many solutions in integers $x, y$. Then by Lemma 1 , there exist $\lambda(x), \kappa(x), \varphi(x) \in \mathbb{Q}[x]$ with $\operatorname{deg} \lambda=\operatorname{deg} \kappa=1$ such that

$$
f_{k}(x)=\varphi(F(\lambda(x))) \quad \text { and } \quad g(x)=\varphi(G(\kappa(x))),
$$

where $F(x), G(x)$ form a standard pair over $\mathbb{Q}$. Theorem 3 implies that

$$
\operatorname{deg} \varphi \in\{1, k+1\} \text {. }
$$

First, suppose that $\operatorname{deg} \varphi(x)=k+1$. Then, by (32), we observe that $\operatorname{deg} F(x)=1$. Thus $f_{k}(x)=\varphi(t(x))$, where $t(x)=F(\lambda(x)) \in \mathbb{Q}[x]$ is a linear polynomial. Clearly, $t^{-1}(x) \in \mathbb{Q}[x]$ is also linear. By (32), we obtain $f_{k}\left(t^{-1}(x)\right)=\varphi\left(t\left(t^{-1}(x)\right)\right)=\varphi(x)$. Hence,

$$
g(x)=\varphi(G(\kappa(x)))=f_{k}\left(t^{-1}(G(\kappa(x)))\right)=f_{k}(h(x)),
$$

where $h(x)=t^{-1}(G(\kappa(x)))$. So, if in this case equation (1) has infinitely many solutions, then $g(x)$ is of the form $f_{k}(h(x))$, where $h \in \mathbb{Q}[x]$ with $\operatorname{deg}(h(x)) \geq 1$.

Next, suppose that $\operatorname{deg} \varphi(x)=1$. Then there exist $\varphi_{1}, \varphi_{0} \in \mathbb{Q}$ with $\varphi_{1} \neq 0$ such that $\varphi(x)=\varphi_{1} x+\varphi_{0}$. We study now the five kinds of standard pairs. In view of our assumptions on $k$ and $\operatorname{deg} g$, it follows that $F(x), G(x)$ cannot form a standard pair of the second kind.

If it is of the third or fourth kind, then $f_{k}\left(\lambda^{-1}(x)\right)=e_{1} D_{\nu}(x, \delta)+e_{0}$ for some $e_{0} \in \mathbb{Q}, e_{1}, \delta \in \mathbb{Q} \backslash\{0\}$, which contradicts Lemma 12 since $\nu=k+1>3$.

Suppose that $F(x), G(x)$ form a standard pair of the fifth kind. Then (32) implies either

(a) $f_{k}(x)=\varphi_{1}\left(\alpha \lambda(x)^{2}-1\right)^{3}+\varphi_{0}$, or

(b) $f_{k}(x)=\varphi_{1}\left(3 \lambda(x)^{4}-4 \lambda(x)^{3}\right)+\varphi_{0}$.

Case (a) implies a nontrivial decomposition of $f_{k}(x)$, which does not exist by Theorem 3 .

In case (b), we have $k=3$. Putting $\lambda(x)=\lambda_{1} x+\lambda_{0}$, we obtain the equation

$$
x^{4}+7 x^{3}+15 x^{2}+10 x=\varphi_{1}\left(3\left(\lambda_{1} x+\lambda_{0}\right)^{4}-4\left(\lambda_{1} x+\lambda_{0}\right)^{3}\right)+\varphi_{0} .
$$


Comparing the coefficients on both sides, we easily get a contradiction. This shows that $F(x), G(x)$ cannot form a standard pair of the fifth kind.

Finally, consider the case when, in (32), $F(x), G(x)$ form a standard pair of the first kind over $\mathbb{Q}$. Then we have either

(a) $f_{k}\left(\lambda^{-1}(x)\right)=\varphi_{1} x^{q}+\varphi_{0}$, or

(b) $f_{k}\left(\lambda^{-1}(x)\right)=\varphi_{1} \alpha x^{r} v(x)^{q}+\varphi_{0}$, where $0 \leq r<q,(r, q)=1$ and $r+\operatorname{deg} v(x)>0$.

The first case (a) is impossible by Lemma 11 since $q=k+1 \geq 4$.

In the second case (b), we have $g(x)=\varphi_{1} \kappa(x)^{q}+\varphi_{0}$. Since $\operatorname{deg} g \geq 3$, we have $q \geq 3$. Put $\lambda^{-1}(x)=\frac{1}{\lambda_{1}} x-\frac{\lambda_{0}}{\lambda_{1}}$. Taking derivatives in relation (b), we obtain

$$
\frac{1}{\lambda_{1}} f_{k}^{\prime}\left(\frac{1}{\lambda_{1}} x-\frac{\lambda_{0}}{\lambda_{1}}\right)=\varphi_{1} \alpha(v(x))^{q-1}\left(r x^{r-1} v(x)+x^{r} q v^{\prime}(x)\right),
$$

which implies that the polynomial $f_{k}^{\prime}(x)$ has a root of multiplicity at least $q-1 \geq 2$. This contradicts Lemma 5 and completes the proof.

\section{Proof of Theorem $1(i)$}

The statement is trivial for $\operatorname{deg} g=0$. In the sequel, let $\operatorname{deg} g=2$. Then there exist rational numbers $a, b, c$ with $a \neq 0$ such that

$$
f_{k}(x)=a y^{2}+b y+c .
$$

Obviously, we can rewrite (35) as

$$
f_{k}(x)+v=a(y+u)^{2},
$$

where $u=\frac{b}{2 a}$ and $v=\frac{b^{2}-4 a c}{4 a}$. Thus, in view of Lemma 3 , it is sufficient to show that the polynomial $f_{k}(x)+s(s \in \mathbb{Q})$ has at least three zeros of odd multiplicity. Assuming the contrary, we can write

$$
f_{k}(x)+s=\left(A x^{2}+B x+C\right)(w(x))^{2},
$$

for some $A, B, C \in \mathbb{Q}, w(x) \in \mathbb{Q}[x]$. Taking derivatives in relation (37), we obtain

$$
f_{k}^{\prime}(x)=w(x)\left((2 A x+B) w(x)+2\left(A x^{2}+B x+C\right) w^{\prime}(x)\right) .
$$

Hence, every root of $w(x)$ is also a root of $f_{k}^{\prime}(x)$. Denote the roots of $w(x)$ by $x_{i}$. For each root $x_{i}$, by (37), we have

$$
f_{k}\left(x_{i}\right)=-s \text {. }
$$

Moreover, the numbers $x_{i}$ are stationary points of the polynomial $f_{k}(x)$. Thus, by Lemma 5 , we get that $f_{k}(x)$ has $\operatorname{deg} w$ equal extrema. Note that $\operatorname{deg} w$ depends on the choice of $A, B, C$ and on the parity of $k$. 
If $k$ is odd (i.e., $\operatorname{deg} f_{k}=k+1$ is even), then (37) implies that either $\operatorname{deg} w=\frac{k-1}{2}($ when $A>0)$ or $\operatorname{deg} w=\frac{k+1}{2}($ when $A=B=0)$. In both cases, it is easy to observe that $f_{k}(x)$ has $k$ extrema, which are $\frac{k-1}{2}$ local maxima and $\frac{k+1}{2}$ local minima. For $k \geq 13$, Lemma 7 yields that $f_{k}(x)$ has three distinct local minima and two distinct local maxima which is a contradiction.

If $k$ is even, then, again by (37), we have $A=0, B>0$ and $\operatorname{deg} w=$ $k / 2$. Furthermore, in this case $f_{k}(x)$ has again $k$ extrema, but now these are $k / 2$ local maxima and $k / 2$ local minima. Again, we get a contradiction with Lemma 7 if $k \geq 13$.

For $k \leq 12$, an easy computation in e.g. Maple shows that the discriminant of $f_{k}(x)+s$, as a polynomial in $s$, has no rational roots and thus $f_{k}(x)+s$ with $3 \leq k \leq 12$ cannot be of the form shown in (37). This completes the proof.

\section{Proof of Theorem 2}

In view of part (i) of Theorem 1 , we may assume that $n>2$. For the first statement in this case, we rewrite (2) as

$$
f_{k}(x)-b=a y^{n} \text {. }
$$

In view of Lemma 3 , it suffices to show that the polynomial on the left hand side of (40) has at least two zeros of multiplicities coprime to $n$. Indirectly, suppose that we have

$$
f_{k}(x)-b=(A x+B)(w(x))^{n},
$$

for some $A, B \in \mathbb{Q}, w(x) \in \mathbb{Q}[x]$. Taking derivatives in relation (41), we obtain

$$
f_{k}^{\prime}(x)=w(x)^{n-1}\left(A w(x)+n(A x+B) w^{\prime}(x)\right) .
$$

Thus, every root of $w(x)$ is a root of $f_{k}^{\prime}(x)$ of multiplicity at least $n-1$, which contradicts Lemma 5 if $n \geq 3$.

Assume now that (2) holds with some $y \neq-1,0,1$. Just as above, one can easily see that $f_{k}(x)-b$ has at least two distinct roots. Hence, by Lemma 2 , the theorem follows.

\section{ACKNowledGements}

The research of the first three authors was supported in part by the Hungarian Academy of Sciences and by the NKFIH grants NK104208 and K115479. The second author was supported by the János Bolyai Scholarship of the Hungarian Academy of Sciences, and by the University of Debrecen. Research of F. L. was supported in part by grants CPRR160325161141 and an A-rated researcher award both from the 
NRF of South Africa and by grant no. 17-02804S of the Czech Granting Agency. Furthermore, this work started during a visit of F. L. at the Mathematical Institute of the University of Debrecen in July 2016. This author thanks that institution for its hospitality and support.

\section{REFERENCES}

1. A. BÉrczes, B. Brindza and L. HAJdu, On the power values of polynomials, Publ. Math. Debrecen 53 (1998), 375-381.

2. F. Beukers, T. N. Shorey and R. Tijdeman, Irreducibility of polynomials and arithmetic progressions with equal products of terms, Number theory in progress, Vol. 1 (Zakopane-Kościelisko, 1997), pp. 11-26, de Gruyter, Berlin, 1999.

3. B. BrindzA, On $S$-integral solutions of the equation $y^{m}=f(x)$, Acta Math. Hungar. 44 (1984), 133-139.

4. Yu. F. BILU and R. F. Tichy, The Diophantine equation $f(x)=g(y)$, Acta Arith. 95 (2000), 261-288.

5. B. Brindza, Yu. F. Bilu, P. Kirschenhofer, Á. Pintér and R. Tichy, Diophantine equations and Bernoulli polynomials, With an appendix by A. Schinzel, Compositio Math. 131 (2002), 173-188.

6. A. Dujella and I. Gusić, Indecomposability of polynomials and related Diophantine equations, Quart. J. Math. 57 (2006), 193-201.

7. P. ERdös and J. L. SElfridge, The product of consecutive integers is never a power, Illinois J. Math. 19 (1975), 292-301.

8. K. Győry, T. KovÁcs, Gy. PÉter and Á. Pintér, Equal values of standard counting polynomials, Publ. Math. Debrecen 84 (2014), 259-277.

9. L. Hajdu, S. LAishram and Sz. Tengely, Power values of sums of products of consecutive integers, Acta Arith. 172 (2016), 333-349.

10. M. Kulkarny and B. Sury, On the Diophantine equation $x(x+1) \ldots(x+$ $m-1)=g(y)$, Indag. Math. 14 (2003), 35-44.

11. R. Lidl, G. Mullen and G. Turnwald, Dickson polynomials, Pitman Monographs and Surveys in Pure and Applied Mathematics 65, Longman Scientific \& Technical, Harlow, 1993.

12. A. Schinzel and R. Tijdeman, On the equation $y^{m}=P(x)$, Acta Arith. 31 (1976), 199-204.

13. R. Tijdeman, Applications of the Gel'fond-Baker method to rational number theory, Topics in Number Theory, Proceedings of the Conference at Debrecen 1974, Colloq. Math. Soc. János Bolyai 13, pp. 399-416, North-Holland, Amsterdam, 1976.

ANDrÁs BAZsó

INSTITUTE OF MATHEMATICS

MTA-DE Research Group "Equations, Functions and Curves" Hungarian Academy of Sciences and University of Debrecen

P.O. Box 12, H-4010 Debrecen, Hungary

Email address: bazsoa@science.unideb.hu 
Attila BéRCZes

Institute of Mathematics

UNIVERSITY OF DEBRECEN

P.O. Box 12, H-4010 Debrecen, Hungary

Email address: berczesa@science.unideb.hu

LAJOS HAJDU

InSTITUTE OF MATHEMATICS

UNIVERSITY OF DEBRECEN

P.O. Box 12, H-4010 Debrecen, Hungary

Email address: hajdul@science.unideb.hu

FLORIAN LUCA

School of Mathematics, Wits University, Private Bag X3, Wits 2050, South Africa,

Max Planck Institute for Mathematics, Bonn, Germany

Department of Mathematics, Faculty of Sciences,

University of Ostrava, 30 Dubna 22, 70103 Ostrava 1, Czech RepubLIC

Email address: florian.luca@wits.ac.za 